\title{
Abnormality Detection for Auxiliary Drive Shafts on Diesel Cars Using Vibration Condition Monitoring
}

\author{
Minoru KONDO \\ Tatsuro TAKASHIGE \\ Drive Systems Laboratory, Vehicle Control Technology Division
}

\begin{abstract}
There are many kinds of rotating components mounted on railway vehicles such as traction motors, generators, and traction gears. The failures of such components sometimes lead to service disruptions and/or accidents. Therefore, it is important to detect their abnormalities at an early stage and prevent their failures. In general, vibration monitoring is an effective abnormality detection method for rotating components. However, detection of the vibration of those components is complicated by vehicle vibration and varied operational status. To address these issues, the authors have proposed an abnormality detection method using vibration octave spectra and machine learning. As a means of verifying the proposed method, engine tests are conducted using auxiliary drive shafts with two simulated abnormalities. The test results indicate that the proposed method enables us to detect them and distinguish between them.
\end{abstract}

Keywords: condition monitoring, abnormality detection, diesel car, machine learning

\section{Introduction}

In a diesel railcar, an auxiliary drive shaft is often utilized to take out power from a diesel engine for driving a generator for auxiliary equipment and a radiator fan. This auxiliary drive shaft is subject to various vibrations and pulsating torques, sometimes causing problems to occur around the shaft. To address this issue, an effective inspection method is desired to prevent accidents by finding the problem in an early stage.

One common method of inspecting rotary equipment utilizes vibration measurements taken during rotation. Since the auxiliary drive shaft rotates while the diesel engine is idling, it is possible to perform vibration measurements during a periodic inspection without operating the car in transit. Therefore, the authors are developing an abnormality detection method that analyzes vibration measured during idling. Two abnormality detection methods are being studied. One method uses a general vibration analyzer and the other method uses an octave band analy$\operatorname{sis}[1,2]$.

In this paper, to verify the abnormality detection method that analyzes the vibration, the authors report on the abnormality simulation test of the auxiliary drive shaft performed on engine bench test equipment. The abnormality simulation was carried out on two kinds of abnormalities associated with auxiliary drive shafts, 1.) abrasion in an attached part and 2.) hardening of a rubber coupling. First, the test equipment and procedure used in the abnormality simulation are described. Then, the vibration measurement method and the analysis method for abnormality detection will be explained. Finally, the measurement results of the vibration and the calculation result of the abnormality are discussed.

\section{Abnormality simulation test method}

\subsection{Test equipment}

The engine bench test equipment is shown in Fig.1. The abnormality simulation test was carried out using a diesel engine on this type of equipment. The output shaft of the engine was attached to the dynamometer. Although it is possible to operate the engine under a load, no load was applied during this test since the test targets only the idling state. On the opposite side of the output shaft of the engine, there is an auxiliary drive shaft for producing power for auxiliary equipment. In this test, since the auxiliary drive shaft must be rotated, it was connected to the engine shaft in the same way as an actual diesel car. In addition, the opposite side of the auxiliary drive shaft was connected to a generator installed in place of the auxiliary equipment drive unit.

Figure 2 shows the auxiliary drive shaft installed on the test equipment. The auxiliary drive shaft consists of a cardan joint, a spline shaft, and a rubber coupling. In an actual vehicle, the cardan joint of the auxiliary drive shaft

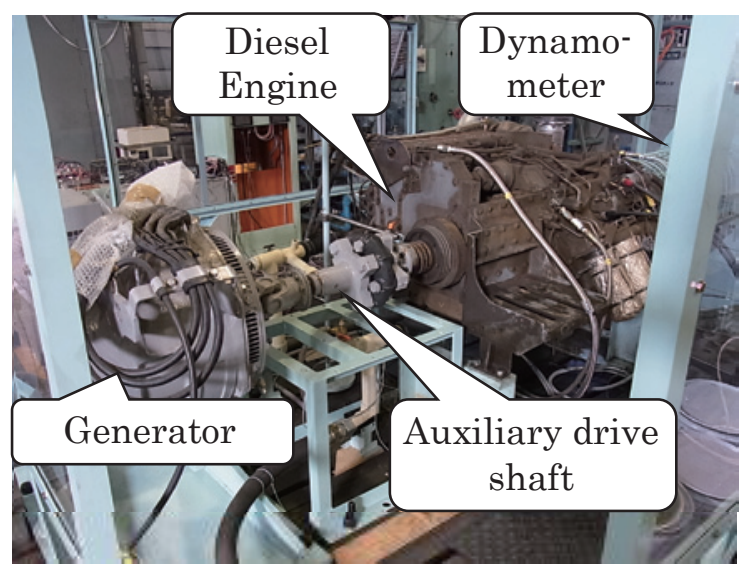

Fig. 1 Engine bench test equipment 


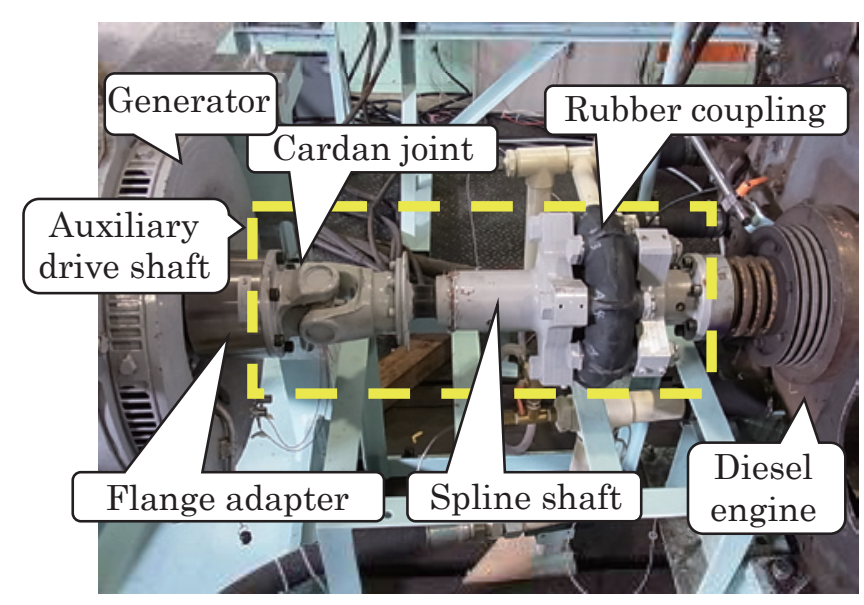

Fig. 2 Auxiliary drive shaft on test equipment

is connected to a flange which is joined to the shaft end of the auxiliary equipment drive unit via a spline-coupling. However, the shaft of the generator used in the test equipment has a tapered end with a keyway. This required us to fabricate a prototype flange adapter with a tapered hole and a keyway, and the cardan joint and the generator were connected via this flange adapter.

\subsection{Abnormal simulation method (loose key)}

One type of failure related to the auxiliary drive shaft occurs when the auxiliary drive shaft gets out of flange joint due to wear at the joint to the auxiliary equipment drive unit. This joint has a spline connection, and when wear occurs, a gap is created in the rotational direction, thus producing abnormal vibration. As a means of simulating this situation in this test equipment, a gap was provided in the rotational direction to generate similar abnormal vibration. This was accomplished by replacing the key for connecting the flange adapter and the generator shaft with one having a width narrower than that of the normal key. While the width of the normal key is $15 \mathrm{~mm}$, the key used for simulating the abnormality is $14 \mathrm{~mm}$, generating a circumferential gap of $1 \mathrm{~mm}$. Figure 3 shows the state of the key joint when simulating abnormality after the circumferential gap has been formed.

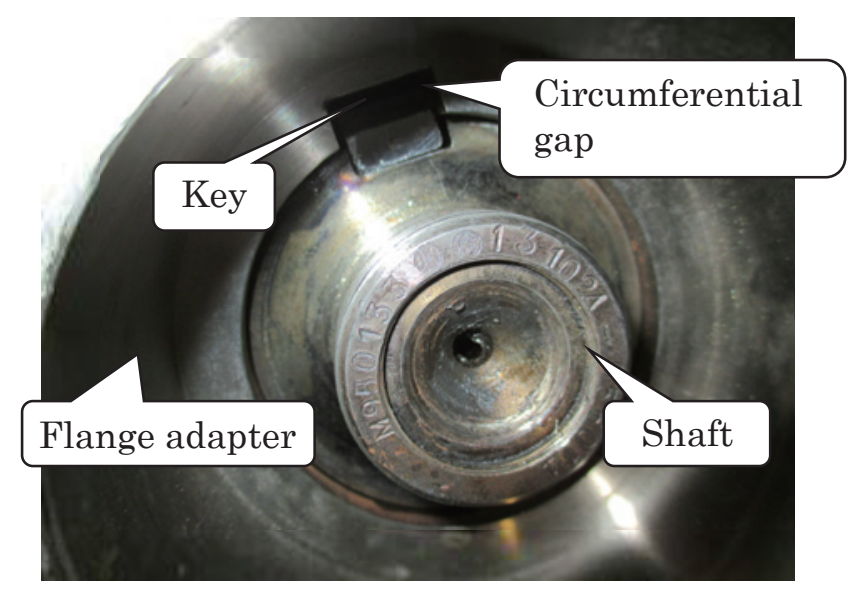

Fig. 3 Simulated abnormality at the key joint

\subsection{Abnormal simulation method (rubber deteriora- tion)}

As previously described, the auxiliary drive shaft has a structure in which a rubber coupling, a spline shaft, and a cardan joint are connected. By using a rubber coupling at the auxiliary drive shaft, torque pulsation of the engine is not directly applied to the auxiliary equipment. On the other hand, since the rubber coupling becomes a spring element, it is assumed that the performance of the rubber coupling influence the torsional vibration. Rubber is known to harden due to age-related deterioration. To simulate this type of deterioration, the rubber coupling used in the simulation was changed to a rubber coupling whose hardness was higher than that of a normal one (See Table 1).

\section{Table 1 Characteristics of rubber couplings}

\begin{tabular}{|c||c|c|}
\hline & $\begin{array}{c}\text { Rubber hardness } \\
\text { (surface) }\end{array}$ & $\begin{array}{c}\text { Torsion spring constant } \\
\text { (calculated value) }\end{array}$ \\
\hline \hline Simulated deterioration & A73 & $29.4 \mathrm{kNm} / \mathrm{rad}$ \\
\hline Normal & A65 & $21.9 \mathrm{kNm} / \mathrm{rad}$ \\
\hline
\end{tabular}

\section{Abnormality detection method}

\subsection{Measurement and analysis using a vibration analyzer}

Inspection of rotary equipment by vibration measurement has been widely performed, and measuring instruments for this purpose are on the market. To determine if we can also apply the conventional method to the auxiliary drive shaft, a portable vibration analyzer (See Fig. 4), which is one of such instruments, was used to perform vibration measurement.

Vibration measurement is performed with this analyzer by attaching a piezoelectric vibration acceleration sensor to the measurement target. Vibration analyzers have several modes. In the vibrometer mode, the measured vibration is processed in real-time, and the root mean square (RMS) value and peak value of vibration acceleration, vibration displacement, vibration speed, and the crest factor

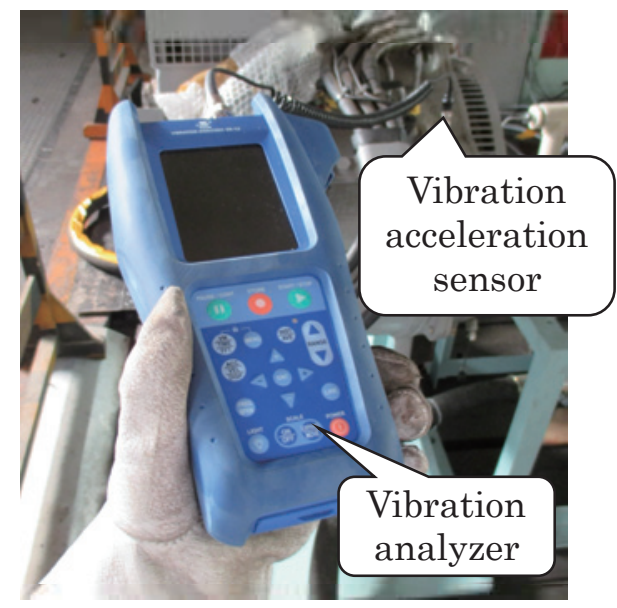

Fig. 4 Rion VA-12 vibration analyzer 
Table 2 Configurations of the vibration analyzer

\begin{tabular}{|c|c|c|}
\hline \multicolumn{2}{|c|}{ Item } & Value \\
\hline \multicolumn{2}{|r|}{ High-pass filter } & $3 \mathrm{~Hz}$ \\
\hline \multicolumn{2}{|r|}{ Low-pass filter } & $5 \mathrm{kHz}$ \\
\hline \multirow[t]{5}{*}{ FFT } & Averaging & Linear average (10 times) \\
\hline & Measurement items & Acceleration, speed, displacement \\
\hline & Frequency span & $200 \mathrm{~Hz}$ \\
\hline & Number of spectral lines & 3200 \\
\hline & Window function & Hanning \\
\hline
\end{tabular}

of vibration acceleration are displayed. In the fast Fourier transform (FFT) analyzer mode, frequency analyses (Fourier transform) of the measured vibration displacement, vibration speed, and vibration acceleration are performed and the results are recorded in a file with use of the vibration analyzer.

For this measurement, the vibrometer mode was used basically to simulate a normal vibration inspection. FFT analyzer mode was applied to perform FFT analyses of vibration displacement, vibration speed, and vibration acceleration for reference and the analyzed results were recorded.

The settings of the vibration analyzer were as shown in Table 2. The measurement range was adjusted as necessary.

\subsection{Measurement and analysis using a condition monitor}

In the periodic inspection by stationary vibration test, it is assumed that a portable vibration analyzer is used as described above. Meanwhile, the authors are developing a condition monitoring system under which a condition monitor is installed on the vehicle and equipment vibration is constantly monitored $[1,2]$. When a vibration acceleration sensor is attached to the auxiliary equipment drive unit, any abnormality in the auxiliary drive shaft should be detected. It is also possible to apply the method used in the condition monitoring system to the stationary vibration test.

Therefore, in this abnormality simulation test, vibration measurement by the condition monitor is also performed at the same time, and the abnormality detection capability of the condition monitoring system under development is evaluated.

The condition monitor is a device for measuring the vibration and recording the result of octave band analysis together with other data such as rotational speed. In the present device, the results of the octave band analysis of the measured vibration acceleration and the average value of the measured voltage are recorded in the internal storage device and the universal serial bus (USB)-connected external storage device. When the condition monitor is turned on, it automatically starts measurement and signal processing. Where the specified measurement value exceeds the preset threshold value, the results are recorded on the output file. To make processing on the computer
Table 3 Specifications of the condition monitor

\begin{tabular}{|c|c|c|}
\hline \multicolumn{2}{|c|}{ Item } & Specification \\
\hline \hline \multirow{3}{*}{ Octave band analysis } & Target channel & Vibration $1 \mathrm{ch} \sim 4 \mathrm{ch}$ \\
\cline { 2 - 3 } & Band width & $1 / 12$ octave \\
\cline { 2 - 3 } & Analysis period & $1 \mathrm{~s}$ \\
\cline { 2 - 3 } & Frequency range & $10 \mathrm{~Hz} \sim 5 \mathrm{kHz}$ \\
\hline Record period & $1 \mathrm{~s}$ \\
\hline Output file format & $\mathrm{CSV}$ \\
\hline
\end{tabular}

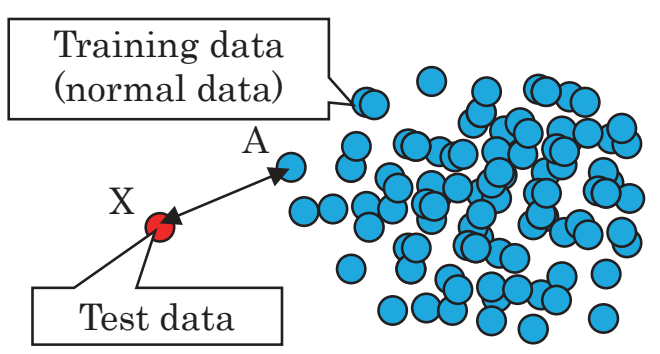

Fig. 5 Schematic of the abnormality calculation

more efficient, the comma-separated values (CSV) text data format was used. Table 3 shows the main software specifications of the condition monitor.

The octave band analysis result obtained by the condition monitor is processed by the diagnostic program to evaluate the abnormality. In the diagnostic program, as shown in Fig. 5, the octave band analysis spectra are transformed into coordinates of points in multi-dimensional space using principal component analysis (PCA).

More specifically, the principal components of an octave band spectrum of measured vibration are the data representing the vibration for abnormality detection, and they become the coordinates of the point in the multidimensional space. Then, according to the concept of the "nearest neighbor" method of machine learning, the training data A closest to the test data X are studied to evaluate the abnormality. The abnormality was defined so that it increases with the distance between $\mathrm{X}$ and $\mathrm{A}$ and has a positive value when that distance is larger than most of the distances between the training data. Details of this abnormality calculation are described in reference [2].

\subsection{Vibration measurement position}

Figure 6 shows the mounting positions of the vibration acceleration sensor. Initially, the vibration acceleration sensor of the vibration analyzer measured the vibration in the vertical direction at the upper part of the generator hanging hole, shown as position A. When taking measurements on a diesel car with a vibration analyzer, a vibration acceleration sensor is attached on the side of the auxiliary equipment drive unit because that location is easy to access, thus simplifying the sensor attachment process. Consequently, position A was selected as a corresponding location.

Next, the vibration acceleration sensor of the condi- 


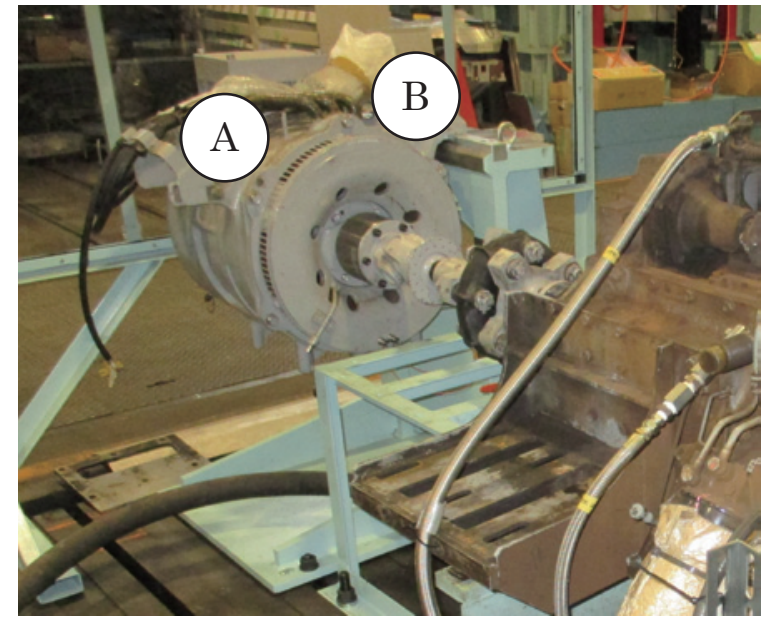

Fig. 6 Vibration measurement position

tion monitor was attached in position B as shown in Fig. 6. Based on experience gained through past research and development projects, it is known that the performance of abnormality detection by the condition monitor does not depend largely on the mounting position of the vibration acceleration sensor, so it is placed in a position where it is relatively easy to attach to the generator with a magnet attachment.

\section{Abnormal simulation test results}

\subsection{Test results using a vibration analyzer}

Following is a description of measurement results and analysis results when using a vibration analyzer in the vibrometer mode. In this mode, measurement values were determined for five measurement items, 1.) the RMS value and 2.) the peak value of the vibration acceleration, 3.) the crest factor, 4.) the vibration speed (RMS value), and 5.) the vibration displacement (equivalent peak-to-peak value). The measurements were acquired under each of the four test conditions shown in Table 4. The operational conditions of the respective tests were all idling.

When the engine started idling after the test conditions were changed, unstable vibration occurred for a while until the parts conformed to each other. Therefore, the measurement was carried out after idling the engine for about 30 minutes. Figure 7 shows the results of vibration speed and vibration displacement extracted from the overall measurement results. In the vibrometer mode, the measured values may fluctuate greatly depending on the time when the measurement is taken. Therefore, the average value of five samples is shown together with the maximum value and the minimum value.

In Fig. 7, "normal 1" and "normal 2" show almost the same measurement values, whereas a significantly higher value was observed in the case of "loose key." From this result, it can be confirmed that the abnormal vibration generated by the presence of the circumferential gap can be detected by measuring the vibration indices such as the vibration speed or vibration displacement. The same tendency was also observed the other vibration indices. However, regarding with the acceleration peak value, the crest factor, and the vibration displacement, the difference between the maximum value and the minimum value was relatively large in the case of the "loose key" tests. This is assumed to be attributable to the occurrence of varied vibrations due to shocks. The vibration speed has the smallest variation among the measurement results, therefore it was considered to be an index suitable for the evaluation of

Table 4 Test conditions

\begin{tabular}{|c|c|c|}
\hline Order & Name & Condition \\
\hline \hline 1 & Normal 1 & Normal condition \\
\hline 2 & Loose key & Circumferential gap at the key joint (Clause 2.2) \\
\hline 3 & Normal 2 & Normal condition \\
\hline 4 & Rubber deterioration & Use of a hardened rubber coupling (Clause 2.3) \\
\hline
\end{tabular}
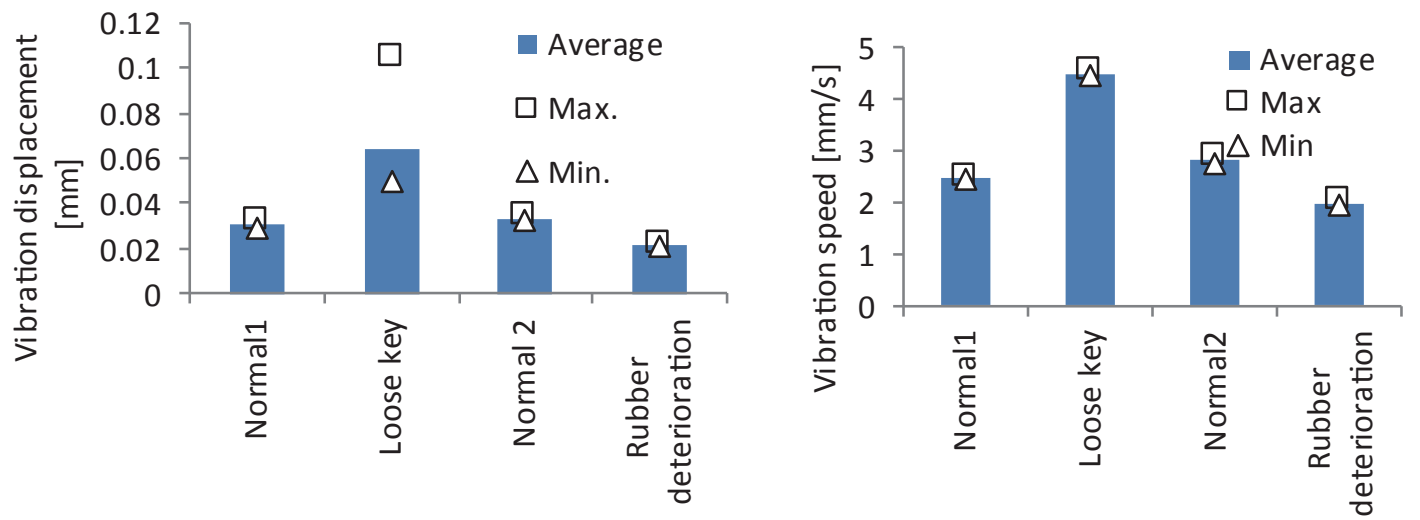

Fig. 7 Measurement results in the vibrometer mode 

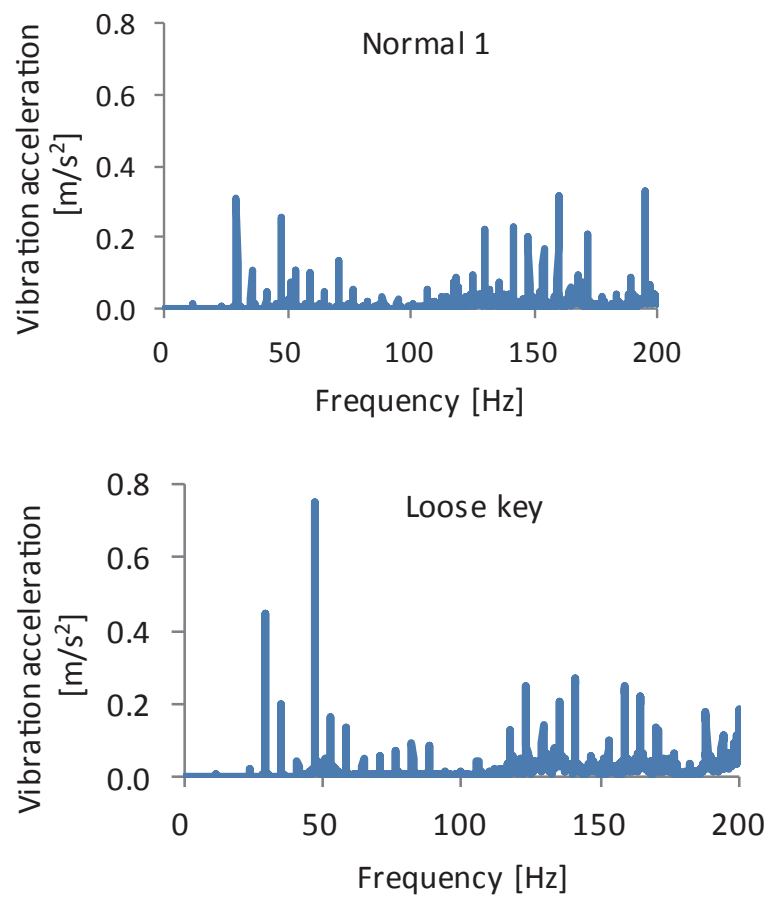
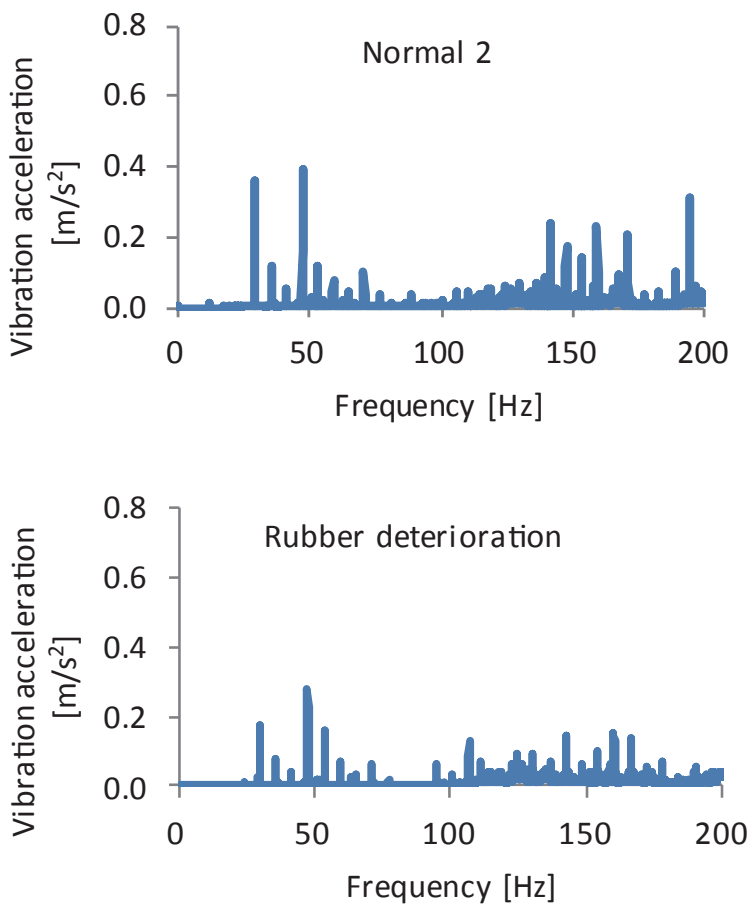

Fig. 8 Measurement results in the FFT mode

the abnormality.

On the other hand, in the case of "rubber deterioration," a value slightly smaller than the normal state is observed. Consequently, it is difficult to detect the rubber deterioration by setting an upper limit value for the measured value in the vibrometer mode. It is thought that one of the reasons why a slightly smaller value is observed is the fact that torsional vibration was suppressed due to rubber hardening.

The following segment deals with measurement results acquired in the FFT mode. In this mode, frequency analysis by FFT was performed on three indices, 1.) vibration acceleration, 2.) vibration speed, and 3.) vibration displacement. The test conditions were the same as those in the vibrometer mode (Table 4), and the measurements were carried out approximately 30 minutes after idling began. The idling speed was approximately 700 to $710 / \mathrm{min}(11.7$ to $11.8 \mathrm{~Hz}$ ). The frequency analysis results of the vibration acceleration are shown in Fig. 8.

The main frequency components observed are approximately the same under each test condition. When comparing the results of the "loose key" state with those of the "normal" states, the $29.4 \mathrm{~Hz}$ component (the 2.5 th order component of the rotational frequency) and the $47 \mathrm{~Hz}$ component (the 4th order component) of the "loose key" state show remarkably high values. Therefore, it is possible, using the frequency analysis results, to distinguish the abnormal vibration produced by the "loose key" from the baseline vibration in the "normal" states. Comparing the results of "rubber deterioration" with the "normal" state, there is no significant difference over all, but it should be noted that the $195 \mathrm{~Hz}$ component observed in the "normal" state is negligible under the condition of "rubber deterioration." Although the reason for such a difference is unknown, it was found that the frequency analysis results of the vibration acceleration clearly shows the change in the vibration due to the rubber deterioration. The frequency analysis results for vibration speed and vibration displacement also produced similar results.

In summary, regarding abnormality detection using the vibration analyzer, it was found that an abnormality due to "loose key" can be detected by measuring the vibration speed. On the other hand, it was found to be difficult to detect abnormalities due to "rubber deterioration" by the same method. However, since the difference in vibration appears in the frequency analysis results, there is a possibility that the rubber deterioration can be evaluated by analyzing those results.

\subsection{Test results using a condition monitor}

The condition monitoring system consists of a condition monitor together with a diagnostic program. The output vibration data of the monitor are used in the diagnostic program for evaluating an abnormality. The following will describe the evaluation of the abnormality by the diagnostic program based on measurement results.

Several algorithms for calculating abnormalities identified by the diagnostic program have been considered, but here the authors use a method [2] based on the "nearest neighbor" method, and as shown in table 5, the values of the parameters are the same as those in the prior examination. However, the extraction of the steady state required for handling the traveling data of the vehicle [1] is not necessary in the case of stationary and idling-only operation. Consequently, the extraction process was disabled by substantially increasing the threshold value. Instead, as a means of excluding data recorded immediately after startup or just before stoppage, a process of extracting data by limiting the range of the engine rotation speed was utilized.

When calculating the abnormality, it is necessary to 
Table 5 Parameters of the diagnostic program

\begin{tabular}{|c|c|}
\hline Parameter & Value \\
\hline \hline Threshold of steady state extraction & 1 \\
\hline Range of engine rotational speed & $600 \sim 800 / \mathrm{min}$ \\
\hline Number of principal components & 6 \\
\hline Number of neighbors & 4 \\
\hline Expected ratio of normal data in training data & $99 \%$ \\
\hline
\end{tabular}

use the vibration data in a normal state as training data. In the current abnormality calculation, all vibration data of "normal 1 " was used in that manner. The test data consisted of vibration data associated with "loose key," "normal 2," and "rubber deterioration" conditions. Vibration data used as test data were extracted from the data of the last 10 minutes in 30 minutes of operation after components were disassembled and reassembled to promote parts conforming well with each other. The histogram of the calculated abnormalities is shown in Fig. 9.

Abnormalities calculated by the diagnostic program have negative values when they are classified as "normal" and positive values when classified as "abnormal." In Fig. 9, although some of the "normal 2" results have positive values, most of them have negative values, and they are generally classified as "normal." In the case of the "loose key" results, abnormality value of all the data was greater than one, and clearly the abnormalities were successfully detected. Regarding the "rubber deterioration" results, $90 \%$ or more of the data have positive abnormalities, and abnormality detection is well-performed here as well.

The reason why a part of "normal 2" is judged to be abnormal is presumably attributable to the fact that vibrations not detected under "normal 1" conditions are actually observed in the case of "normal 2." Since the operational specifics under normal conditions also change for various reasons, there is a high possibility that the data of "normal 1 " testing do not cover all normal conditions. However, when applying this method to commercial vehicles, it is reasonably expected that a great deal of training data covering a large variety of normal conditions are acquired over a long period of time.

The following explains how, by using the abnormal

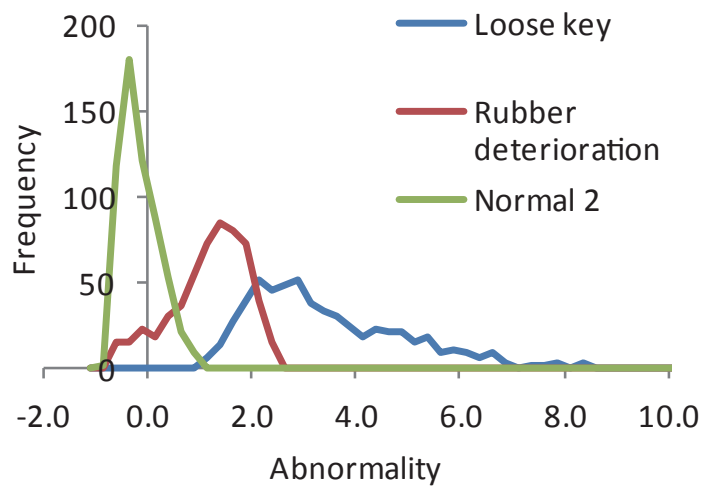

Fig. 9 Histogram of abnormality data as the training data, the type of abnormality can be diagnosed. That determination begins with the abnormal data of "loose key" and "rubber deterioration" being divided into the first half and the latter half of each condition, becoming "loose key 1 " and "loose key 2," and "rubber deterioration 1 " and "rubber deterioration 2," respectively. Then, for example, to judge whether or not test data corresponds to "loose key," "loose key 1 " is used as training data and the correspondence to the training data is calculated by multiplying the abnormality of the test data by negative one. Since the abnormality originally indicates the degree of non-correspondence to the training data, the value multiplied by negative one is the degree of correspondence to the training data. The calculation results of the correspondence with the "loose key 1 " training data are shown in the upper graph of Fig. 10 and the correspondence with the "rubber deterioration 1 " training data is shown in the lower graph of Fig. 10.

As seen in the upper graph of Fig. 10, since most of the data corresponding to "loose key 2 " data have a positive value, the presence of a gap can be inferred. On the other hand, it is possible to judge that there is no gap in "rubber deterioration 2 " since the data corresponding to the "rubber deterioration 2 " data have negative values. Similarly, from the lower graph of Fig. 10, it can be judged that the "loose key 2 " data do not correspond to rubber deterioration and "rubber deterioration 2 " data do correspond to rubber de-

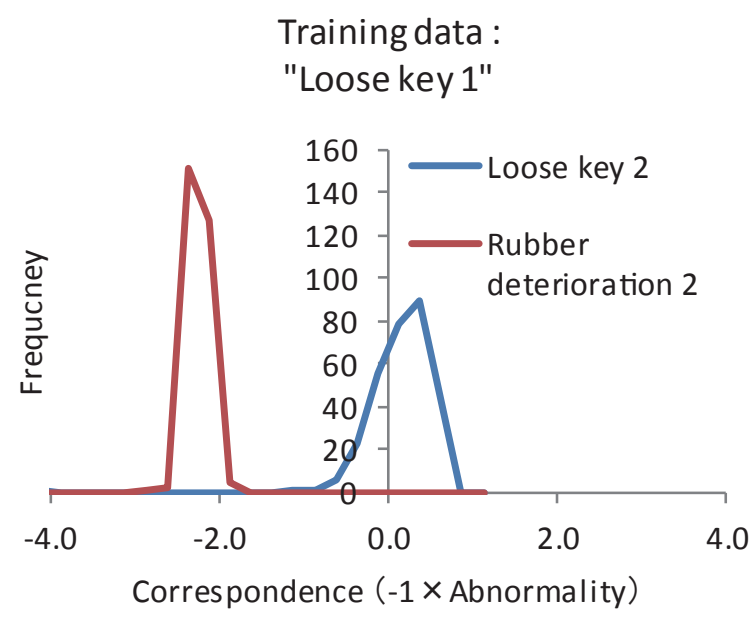

Training data :

"Rubber deterioration 1"

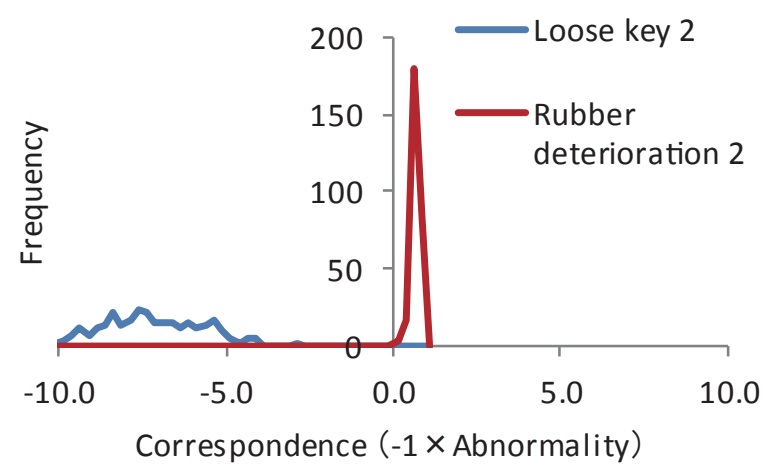

Fig. 10 Histogram of correspondence 
terioration. In this way, it was confirmed that by using abnormal data as training data, it is possible to diagnose the type of abnormality.

\section{Conclusions}

In order to develop inspection methods for the auxiliary equipment drive systems utilizing vibration measurement, the authors conducted an abnormality simulation test of the auxiliary drive shaft in engine bench test equipment and verified whether the abnormalities can be detected by the method proposed.

As a result, it was confirmed that changes in vibration produced by abnormality simulation can be detected both by the method using the vibration analyzer and the method using the condition monitor under development. In the case of the condition monitoring system, it was concluded that there is a possibility that abnormality type can be diagnosed by using past abnormal vibration data as training data.

\section{References}

[1] Kondo, M., Manabe, S., Takashige, T., and Kanno H., "Abnormality detection of traction diesel engines using vibration analysis in octave bands," RTRI Report, Vol. 29, No. 9, pp. 17-22, 2015 (in Japanese).

[2] Kondo, M., Takashige, T., Manabe, S., and Kanno, H., "Abnormality detection in a contaminated diesel engine by a vibration monitoring method," RTRI Report, Vol. 30, No. 4, pp. 47-52, 2016 (in Japanese).

\section{Authors}

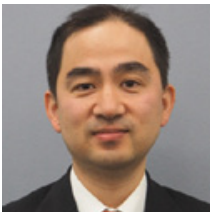

Minoru KONDO, Dr. Eng.

Senior Researcher, Drive systems Laboratory, Vehicle Control Technology Division Research Areas: Traction Motor, Condition Monitoring

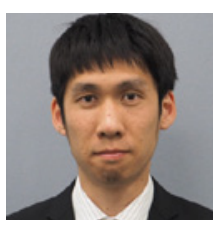

Tatsuro TAKASHIGE

Assistant Senior Researcher, Drive systems Laboratory, Vehicle Control Technology Division

Research Areas: Diesel Engine 